
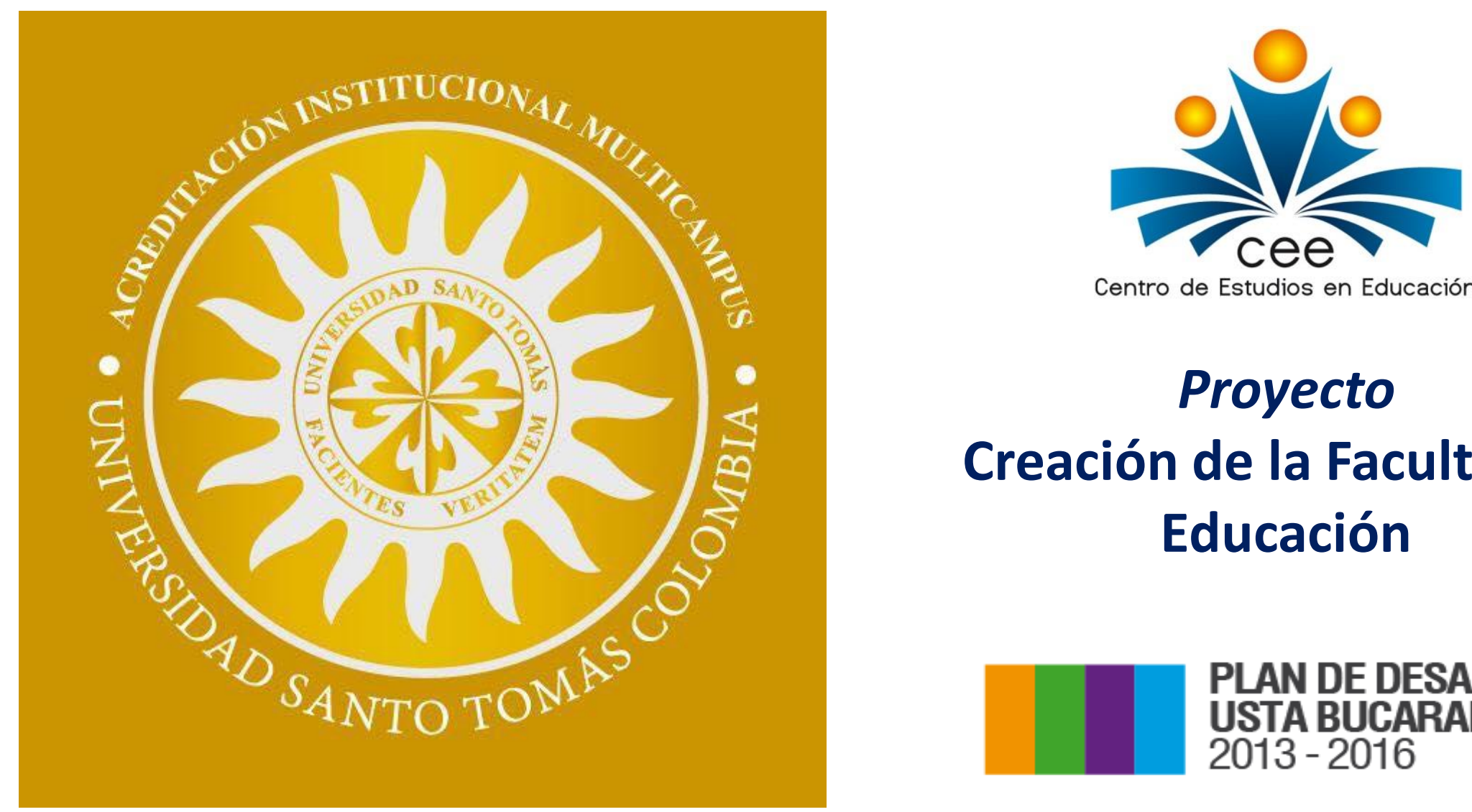

Centro de Estudios en Educación

\title{
Proyecto \\ Creación de la Facultad de Educación
}

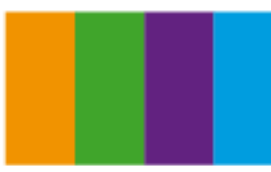

PLAN DE DESARROLLO USTA BUCARAMANGA 2013-2016 
En el camino de los retos de la educación...

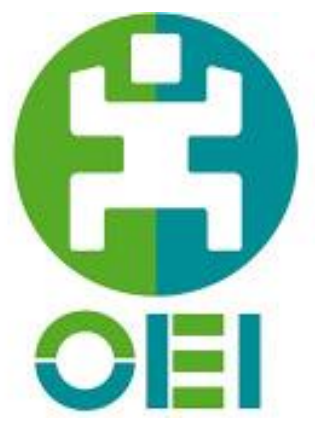

Organización de Estados Iberoamericanos

"Metas Educativas 2021"

$\checkmark$ Formar profesores para las etapas iniciales de la vida, que desempeñen sus carreras con infantes y adolescentes en condiciones de pobreza y vulnerabilidad.

$\checkmark$ El reconocimiento de la carrera y un estatuto docente por la labor desempeñada. 


\section{En el camino de los retos de la educación...}

$\checkmark$ Establecer expectativas claras para los maestros.

$\checkmark$ Atraer a los mejores para la enseñanza.

$\checkmark$ Preparar a los profesores con formación útil y experiencia.

$\checkmark$ Relacionar las habilidades de los docentes con las necesidades de los estudiantes.

$\checkmark$ Liderar a docentes mediante sólidos directores.

$\checkmark$ Apoyar a los docentes para mejorar la instrucción.

$\checkmark$ Motivar a los docentes para el desempeño"

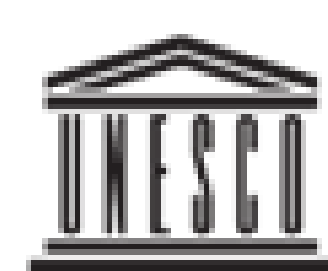

Organización : de las Naciones Unidas = para la Educación, la Ciencia y la Cultura

(OREALC \& UNESCO, $2011: 17$ )

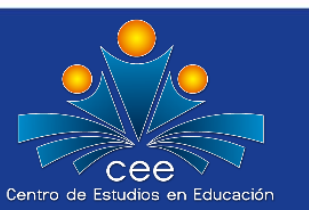


En el camino de los retos de la educación...

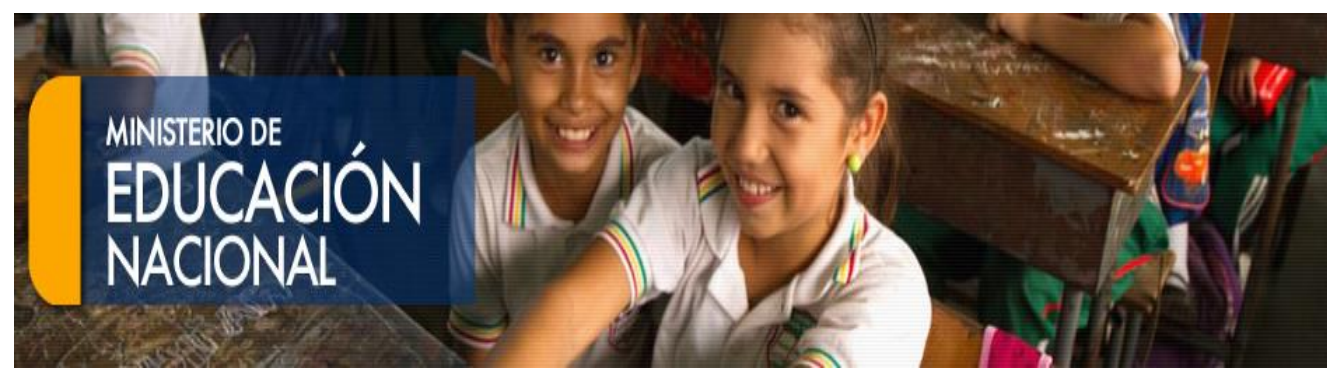

COLOMBIA LA MÁS EDUCADA EN 2025

Gobierno ofrece becas para estudiantes que quieran ser profesores

'Ser pilo paga' dará 1.000 becas adicionales para los bachilleres que opten por las licenciaturas.

Septiembre 2015

ICETEX Maestrías con créditos condonables.

- Ley General de Educación 115 de 1994.

- Ley 1279 de 2002 ascenso en el escalafón docente por formación pregrado y posgrado

- Sistema Colombiano de Formación de Educadores y Lineamientos de Política 2014.

- Decreto 1075 de 2015. Único reglamentario del sector de la Educación.

- Decreto 2450 de 17 Dic 2015 y Resolución 020413 Febrero 2016: Reglamentan condiciones de calidad para creación, renovación o modificación de Licenciaturas.

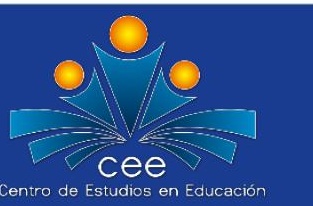




\section{Problemas a intervenir para lograr este reto:}

\section{Cobertura}

Calidad

Permanencia
Escasa formación de docentes en las diversas áreas de conocimiento Ej. Ingles, Matemática, Química, Informática y Tecnología, entre otras.

Poca apropiación de estratégicas, técnicas, y acciones pedagógicas y didácticas para el desarrollo de los procesos enseñanza - aprendizaje y de evaluación que contribuyan al logro de los estándares básicos de Competencias en los estudiantes (básica, media y superior).

Falta de formación docente para asumir los retos de la educación inclusiva diferencial.

Escasa comprensión de los procesos educativos para la etnoeducación y poblaciones vulnerables (posconflicto). 


\section{MISIÓN}

La Facultad de Educación de la Universidad Santo Tomás Bucaramanga, tiene la misión de generar conocimiento y formar profesionales en Educación y Pedagogía para impactar la educación regional y nacional, contribuyendo así en la construcción de sociedades democráticas donde prime la ética del género humano y una visión socio-ecológica. 


\section{Proyecto: Creación de la Facultad de Educación}

\section{VISIÓN}

En el año 2025 la Facultad de Educación de la Universidad Santo Tomás - Bucaramanga, se consolidará como la unidad académica orientadora del desarrollo de los procesos pedagógicos institucionales y, en el entorno regional y nacional será reconocida por ofrecer programas de pregrado, posgrado y educación continua en el ámbito de la educación y pedagogía con excelencia académica y proyección internacional, así como por el desarrollo de procesos de investigación e intervención educativa de relevancia en estos ámbitos. 


\section{MARCO NORMATIVO INSTITUCIONAL}

Estatuto Orgánico de la Universidad Santo Tomás (2010)

ARTíCULO 72.- División es la unidad académico-administrativa, que interrelaciona varias facultades de áreas afines del conocimiento.

ARTíCULO 77.- Las Facultades son unidades académicas conformadas orgánicamente para impartir la docencia, propiciar la investigación en programas específicos de carácter profesional y de formación avanzada, fomentar la interdisciplinariedad y prestar servicio a la comunidad.

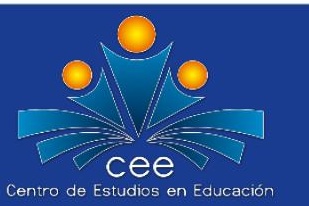




\section{USTA COLOMBIA}

\section{Vicerrectoría de Educación Abierta y a Distancia - VUAD}

FACULTAD DE EDUCACIÓN
Decano de facultad

Director por: Programas de pregrado (1 1)

Director por: Programas de posgrado (5)

Director: Educación Continua (6)

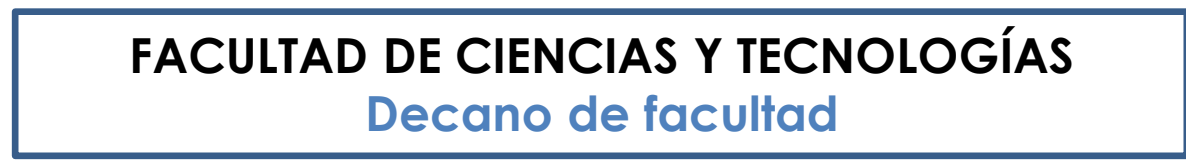

$\checkmark \quad$ Director por: Programas de pregrado (6)

$\checkmark \quad$ Director por: Programas de posgrado (4)

$\checkmark$ Director: Educación Continua (16)

\section{USTA Bogotó}

\begin{tabular}{|c|c|}
\hline \multicolumn{2}{|c|}{ DIVISIÓN DE FILOSOFÍA Y TEOLOGÍA } \\
\hline $\begin{array}{c}\text { FACULTAD DE TEOLOGIA } \\
\text { Decano de facultad }\end{array}$ \\
\hline$\checkmark \quad$ Teología \\
USTA Tunja \\
\hline $\begin{array}{c}\text { FACULTAD DE FILOSC } \\
\text { Decano de fa }\end{array}$ \\
\hline $\begin{array}{c}\text { Centro de Estudios Educativos: Enrique Lacordaire y Ler } \\
\text { Maestria en Pedagógia }\end{array}$ \\
\hline Unidad de Desarrollo Curricular y Formación Docente \\
Educación continua
\end{tabular}

Unidad de Desarrollo Curricular y Formación Docente

Educación Continua

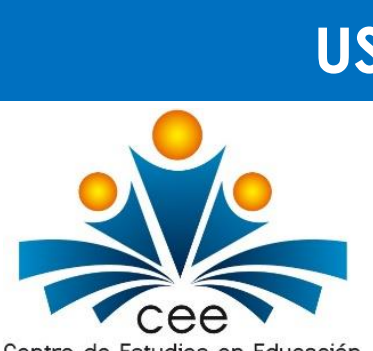

USTA Bucaramanga

Maestria en Educación Ambiental

Programa de Formación Docente Educación Continua

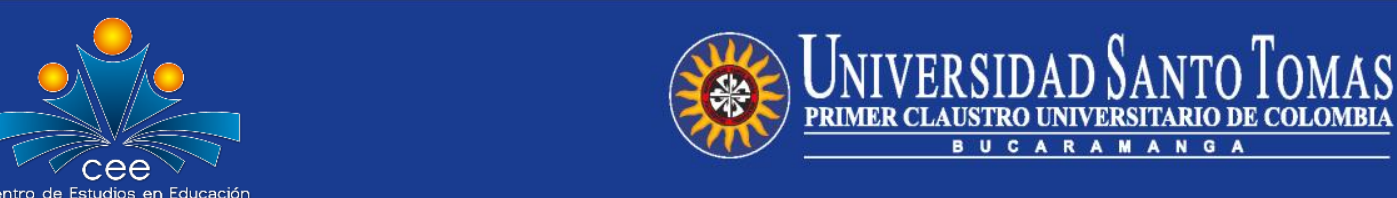




\section{Proyección de la formación docente en la Región y el País}

\section{Comité}

Departamental del Educación Superior
ASCOFADE

Presidencia Capítulo

NorOriente

Vicepresidencia Nacional
Comité Territorial de Formación Docente del Departamento de Santander

Comité Territorial de Formación Docente del Municipio de Girón
Comité Territorial de Formación Docente del Municipio de Floridablanca

Comité Territorial de Formación Docente del Municipio de Piedecuesta 
Facultad de Ciencias Humanas: Escuela de Educación Licenciatura en Educación Básica con énfasis en Ciencias Naturales

y Educación Ambiental.

Licenciatura en Educación Básica con énfasis en Lengua

Castellana.

Maestría en Pedagogía.

Facultad de Ciencias Humanas: Escuela de Artes y Música Licenciatura en Música.

Facultad de Ciencias Humanas: Escuela de Idiomas

Licenciatura en Inglés.

Licenciatura en Español y Literatura.

Maestría en Semiótica

Maestría en Didáctica de la Lengua.

Facultad de Ciencias Humanas: Escuela de Matemáticas Licenciatura en Matemáticas.

Maestria en educación matemática.

\section{Facultad de Educación}

Licenciatura en Lengua Castellana e Inglés

Licenciatura en Educación Física, Recreación y Deporte.

Técnico Profesional en Fútbol.

Especialización en Docencia Universitaria.

Especialización en Ludopedagogía de la Educación Motríz.

Maestría en Educación.
Facultad de Ciencias Sociales, Humanidades y Artes

Licenciatura en Educación Preescolar.

UNAB Virtual

Maestria en E-learning.

Especialización en educación con nuevas tecnologías.

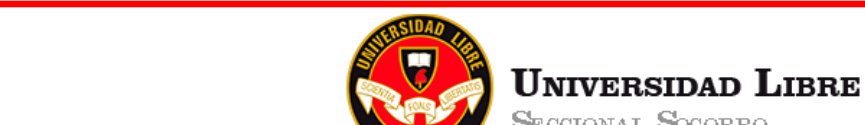

Segccional Socorro

\section{Facultad de Ciencias de la Educación}

- Licenciatura en Ciencias Básicas de la Educación con énfasis en Matemáticas

- Licenciatura en Ciencias Básicas de la Educación con énfasis en Humanidades y Lenguas.

- Licenciatura en Ciencias Básicas de la Educación con énfasis en Ciencias Sociales.

- Licenciatura en Ciencias Básicas de la Educación con énfasis en Ciencias Naturales y Educación Ambiental.

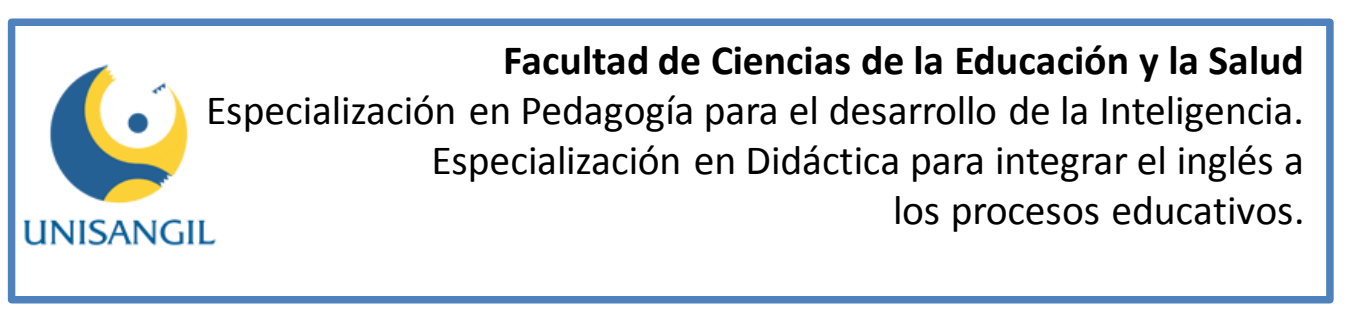

Facultad de Educación

Creada en Julio de 2015

Campus Virtual UDES

Iniversidad de samtander

Maestria en Gestión de la Tecnología Educativa.

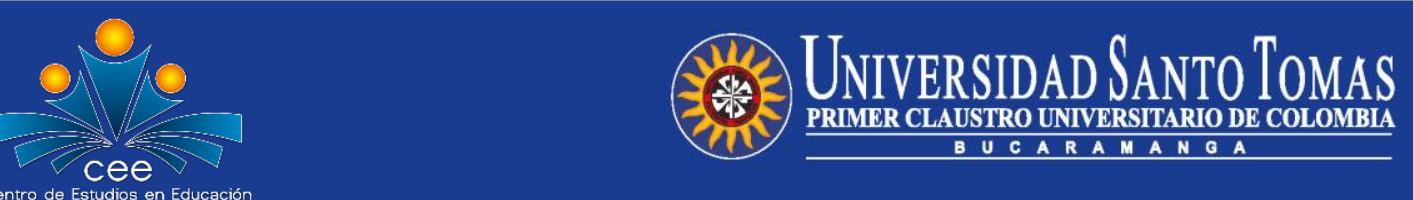


- $\quad$ Licenciatura en Artes Plásticas y Visuales.

- Licenciatura en Biología con énfasis en Educación Ambiental.

- Licenciatura en Educación Básica con énfasis en Humanidades y Lengua Castellana.

- Licenciatura en Educación Básica con énfasis en Matemáticas.

- Licenciatura en Educación Preescolar.

- Licenciatura en Filosofía y Educación Religiosa.

- Licenciatura en Filosofía, Pensamiento Político y Económico.

- Licenciatura en Lengua Castellana y Literatura.

- Licenciatura en Lengua Extranjera Inglés.

- Licenciatura en Informática Educativa.

- Licenciatura en Teología.

- Especialización en Pedagogía para la Educación Superior.

- Especialización en Evaluación Educativa.

- Maestria en Educación.

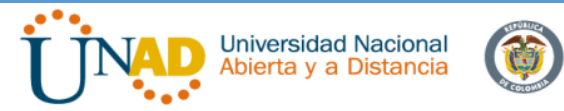

Licenciatura en Etnoeducación.

Licenciatura en Filosofía.

Licenciatura en Inglés como Lengua Extranjera.

Licenciatura en Matemáticas.

Licenciatura en Pedagogía Infantil. 


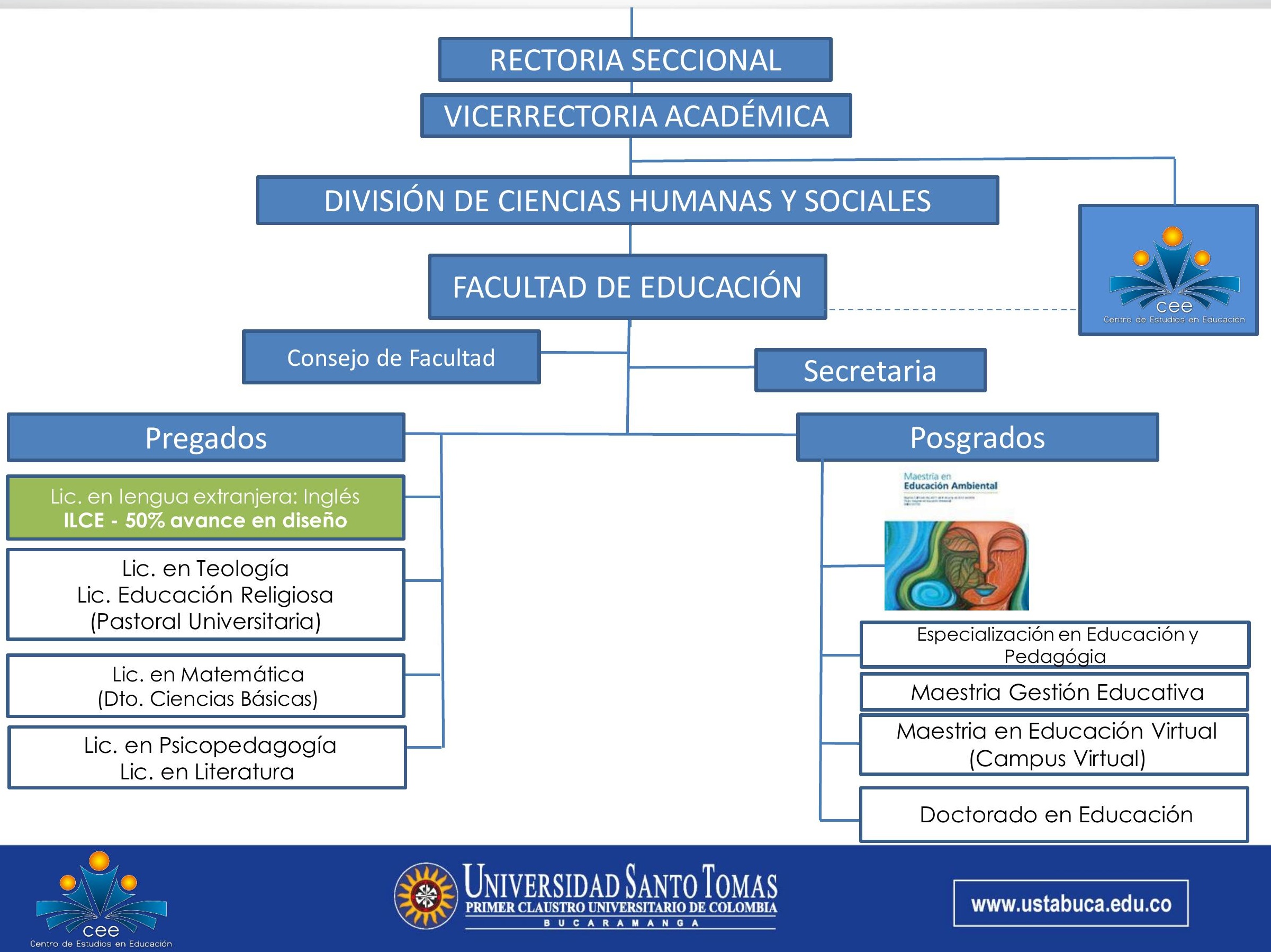




\section{División de Ciencias Sociales y Humanas}

\section{Un modo de pensar y actuar metodológicamente: Complejidad y Transdisciplinariedad}

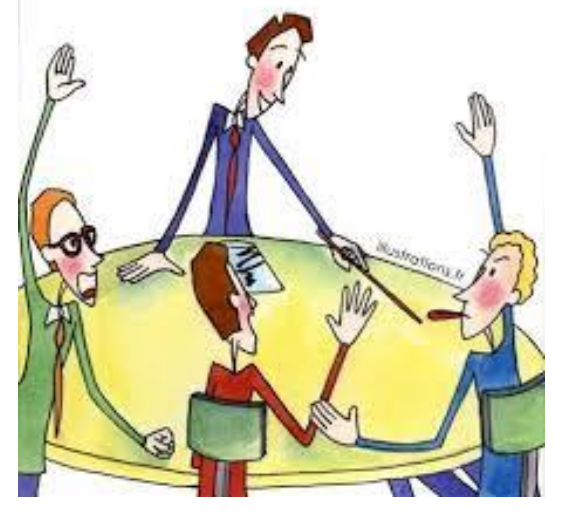

"la era de las disciplinas puede no haber terminado todavía, pero otras formas de organizar el conocimiento están emergiendo en un nivel local y algunas veces regional y supranacional" (UNESCO y Foro Consultivo, 201 1:198). lo cual exige enfoques y metodologías interdisciplinarias, multidisciplinarias y transdisciplinarias.

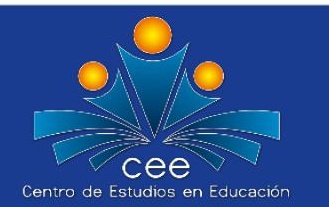




\section{GRAN ÁREA DEL CONOCIMIENTO}

\begin{tabular}{c|c}
\hline 1. & CIENCIAS AGRÍCOLAS \\
\hline 2. & CIENCIAS MÉDICAS Y DE LA SALUD \\
\hline 3. & CIENCIAS NATURALES \\
\hline 4. & CIENCIAS SOCIALES \\
\hline 5. & HUMANIDADES \\
\hline 6. & INGENIERÍA Y TECNOLOGÍA \\
\hline
\end{tabular}




\begin{tabular}{|c|c|c|}
\hline \multirow{9}{*}{ 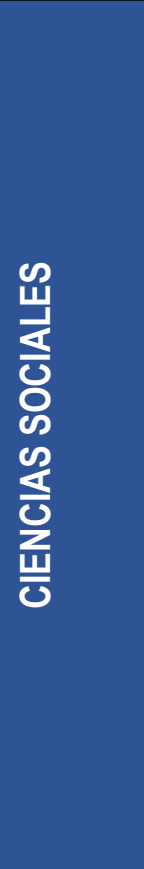 } & CIENCIA POLÍTICA & Administración pública, Ciencias políticas, Teoría organizacional. \\
\hline & CIENCIAS DE LA EDUCACIÓN & Educación Especial, Educación General . \\
\hline & DERECHO & Derecho, Penal. \\
\hline & ECONOMÍA Y NEGOCIOS & Econometría, Economía, Negocios y Management, Relaciones Industriales. \\
\hline & GEOGRAFÍA SOCIAL Y ECONÓMICA & $\begin{array}{l}\text { Ciencias Ambientales (Aspectos Sociales), Estudios Urbanos (Planificación y } \\
\text { desarrollo), Geografía Económica y Cultural, Planificación del Transporte y } \\
\text { Aspectos Sociales del Transporte. }\end{array}$ \\
\hline & OTRAS CIENCIAS SOCIALES & Ciencias Sociales, Interdisiplinaria, Otras Ciencias Sociales. \\
\hline & PERIODISMO Y COMUNICACIONES & $\begin{array}{l}\text { Bibliotecología, Ciencias de la Información (Aspectos Sociales), Medios y } \\
\text { Comunicación Social, Periodismo. }\end{array}$ \\
\hline & PSICOLOGÍA & Psicología \\
\hline & SOCIOLOGÍA & $\begin{array}{l}\text { Antropología, Demografía, Etnografía, Sociología, Temas especiales (Estudios de } \\
\text { género, temas sociales, Estudios de familia, Trabajo Social). }\end{array}$ \\
\hline NÁREA & ÁREA & ESPECIALIDAD \\
\hline \multirow{5}{*}{$\frac{\text { 出 }}{\frac{\text { 号 }}{2}}$} & ARTE & $\begin{array}{l}\text { Arquitectura y Urbanismo, Arte, Artes de la representación (Musicología, Ciencias } \\
\text { del teatro, Dramaturgia), Diseño Arquitectónico, Diseño Industrial y Otros Diseños, } \\
\text { Estudios del Folclor, Estudios de cine, radio, televisión, Historia del Arte. }\end{array}$ \\
\hline & HISTORIA Y ARQUEOLOGÍA & Arqueología, Historia, Historia de Colombia. \\
\hline & IDIOMAS Y LITERATURA & $\begin{array}{l}\text { Estudios Generales del Lenguaje, Estudios Literarios, Idiomas Específicos, } \\
\text { Lingüistica, Literatura Específica, Teoría Literaria. }\end{array}$ \\
\hline & OTRAS HUMANIDADES & Filosofía, Otras Humanidades, Teología. \\
\hline & OTRAS HISTORIAS & Historia de la Ciencia y la Tecnología, Otras Historias Especializadas \\
\hline
\end{tabular}

Fuente: elaboración propia con base en

http://www.colciencias.gov.co/sites/default/files/ckeditor files/files/Manual CvLAC.pdf

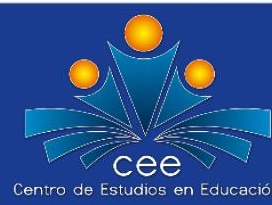




\section{Otras facultades que podrían integrar ésta División}

Dependiendo de los intereses institucionales de orden misional, filosófico y organizacional, así como de los estudios de factibilidad, contexto, pertinencia y estudio del mercado.

$>$ Gobierno y relaciones internacionales

$>$ Geografía social y económica

$>$ Trabajo social (interdisciplinaria)

$>$ Periodismo y comunicaciones

$>$ Archivística

$>$ Bibliotecología

$>$ Psicología

> Sociología

$>$ Economía
$>$ Antropología

$>$ Arte

$>$ Historia y arqueología

$>$ Gestión cultural

$>$ Idiomas y literatura

$>$ Filología

$>$ Filosofía

$>$ Teología

$>$ Derecho

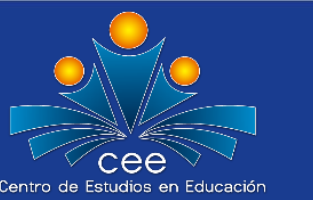




\section{División de Ciencias Sociales y Humanas}

\section{Objetivo General}

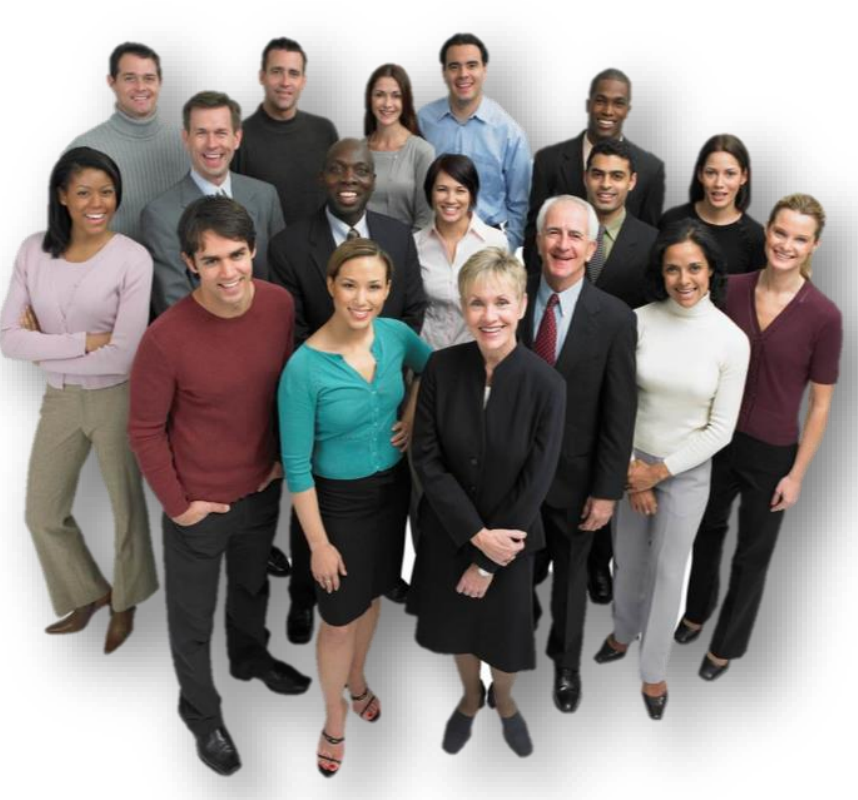

Constituir una unidad académicoadministrativa dedicada a la reflexión transdiciplinaria de las Ciencias Sociales y Humanas, que agencie una propuesta formativa integral e identitaria del profesional tomasino capaz de responder a las problemáticas actuales propios de ámbito de estudio.

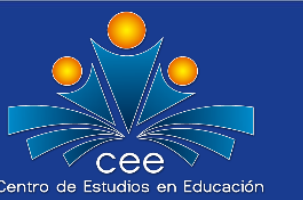




\section{Objetivos Específicos}

> Implementar de manera gradual programas de formación profesional y posgradual en el campo de las Ciencias Sociales y Humanas, a partir de los intereses investigativos e institucionales, como respuesta a la exigencia regional, nacional e internacional de cualificar profesionales en esta gran área del conocimiento.

> Propiciar la reflexión socio humanística en torno a las exigencias morales, éticas y políticas, de las preocupaciones sociales contemporáneas, a fin que le den sentido a la comprensión y responsabilidad del papel desempeñado por los seres humanos como especie inteligente y creadora de las condiciones de cambio socioecológico, que permitan la resolución de problemáticas para el desarrollo regional, la política pública y la cultura.

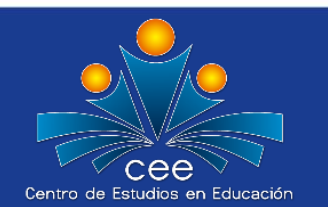


CABEZA, O. (2014). ANTECEDENTE: ESTUDIO ORGANIZACIÓN DE LA USTA EN CUANTO: Facultad de Educación, Vicerrectoría de Universidad Abierta y a Distancia - VUAD. Bucaramanga: Centro de Estudios en Educación.

CICS/UNESCO. (2013). Informe mundial sobre las ciencias sociales 2013 - Cambios ambientales globales. Paris (Francia): Ediciones OCDE y Ediciones UNESCO.

COLCIENCIAS. (2014). Ciencias, Tecnologías e Innovación de las áreas Sociales y Humanas. Recuperado de:

http://www.colciencias.gov.co/programa_estrategia/ciencias-tecnolog-e-innovaci-n-de-las-reas-

sociales-y-humanas Fecha de consulta: 02/10/2014

CONGRESO DE LA REPÚBLICA. (2009). Ley 1286: Por la cual se modifica la Ley 29 de 1990, se transforma a

Colciencias en Departamento Administrativo, se fortalece el Sistema Nacional de Ciencia, Tecnología

e Innovación en Colombia y se dictan otras disposiciones. Colombia.

CONSEJO DE FUNDADORES. (2010). Estatuto Orgánico. Bogotá: USTA

Departamento Administrativo de la Función Pública. (2014). Decreto 173 del 7 de Febrero. República de

Colombia. Recuperado de http://aspucol.org/sitio/wp-content/uploads/2014/02/DECRETO-173-DEL-07DE-FEBRERO-DE-2014.pdf

Departamento Administrativo de la Función Pública. (2014). Decreto 171 del 7 de Febrero. República de Colombia. Recuperado de http://www.mineducacion.gov.co/1621/articles253999_Decreto_171_de_2014.pdf

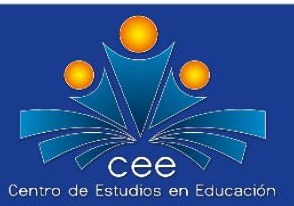


ISSC \& UNESCO. (2013). World Social Science Report 2013, Changing Global Environments. Paris: OECD Publishing and UNESCO Publishing.

LÓPEZ, B. (2012). Sistema Institucional de Formación Permanente del Profesorado, Lineamientos. Bucaramanga: Universidad Santo Tomás.

LÓPEZ, B. (2011). Centro de Estudios en Educación, Estructura y Horizonte. Bucaramanga: Universidad Santo Tomás.

Ministerio de Educación Nacional. (2012). Política y Sistema Colombiano de Formación y Desarrollo Profesional de Educadores.

Colombia.

Ministerio de Educación Nacional. (1979). Decreto 2277 del 14 de Septiembre. República de Colombia. Recuperado de

http://www.mineducacion.gov.co/1621/articles-103879_archivo_pdf.pdf

OCDE \& Banco Mundial. (2012). La educación Superior en Colombia.

OREALC \&UNESCO. (2011). Nueva Agenda de Políticas Docentes en América Latina y el Caribe

OEl. (2010). Metas Educativas 2021: La educación que queremos para la generación de los bicentenarios. Madrid: Cudipal.

Recuperado de: http://www.oei.es/metas2021.pdf

República de Colombia. (2002). Decreto 1278 de Junio 19. Colombia. Recuperado de

http://www.mineducacion.gov.co/1621/articles-86102 archivo pdf.pdf

República de Colombia. (2002). Decreto 1279 de Junio 19. Colombia. Recuperado de

http://www.mineducacion.gov.co/1621/articles-86434_Archivo_pdf.pdf

Richard, M. (2011). Estudio comparado sobre la formación y antecedentes académicos de los docentes en seis naciones. PREAL.

UNESCO y Foro Consultivo. (2011). Informe mundial sobre las ciencias sociales: Las brechas del conocimiento. México: UNESCO.

UNESCO. (2010). Informe mundial sobre las ciencias sociales. Divisorias del conocimiento. Resumen. Francia: UNESCO.

USTA. (2010). Modelo Educativo Pedagógico. Bogotá: Departamento de Publicaciones USTA.

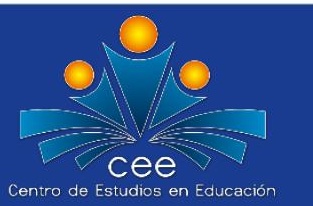




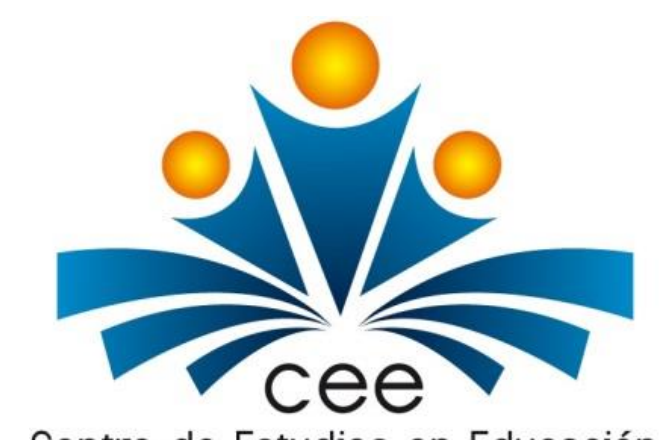

Centro de Estudios en Educación

Responsables del Proyecto:

Nelly Milady López Rodríguez Ph.D. - Oscar Javier Cabeza Herrera M.Sc.

Consejo Directivo

Acta No. 083 de febrero 8 de 2016

Consejo Académico Particular

Acta No. 214 de febrero 8 de 2016

Consejo del Centro de Estudios en Educación

Acta 149 Oct. 24/14 - Acta 167 Nov. 19/15 